\title{
Evaluating the effects of sodium glucose co-transporter -2 inhibitors from a renin-angiotensin-aldosterone system perspective in patients infected with COVID-19: contextualizing findings from the dapagliflozin in respiratory failure in patients with COVID-19 study
}

\author{
Diala Alhaj Moustafa, ${ }^{1,2}$ Zainab Imran ${ }^{1,2} \cdot$ Roua Ismail $^{1,2} \cdot$ Menatallah Rayan $^{1,2} \cdot$ Alain-Pierre Gadeau $^{3}$. \\ Hussein Eldassouki ${ }^{4} \cdot$ Nabeel Abdulrahman ${ }^{1,2,5} \cdot$ Fatima Mraiche $^{1,2}$ (D)
}

Received: 17 September 2021 / Accepted: 20 January 2022 / Published online: 31 January 2022

(c) The Author(s), under exclusive licence to Springer Nature B.V. 2022

\begin{abstract}
Numerous studies demonstrate parallels between CVD, type 2 diabetes mellitus (T2DM) and COVID-19 pathology, which accentuate pre-existing complications in patients infected with COVID-19 and potentially exacerbate the infection course. Antidiabetic drugs such as sodium-glucose transporter-2 (SGLT-2) inhibitors have garnered substantial attention recently due to their efficacy in reducing the severity of cardiorenal disease. The effect of SGLT-2 inhibitors in patients with COVID19 remains unclear particularly since SGLT-2 inhibitors contribute to altering the RAAS cascade activity, which includes ACE-2, the major cell entry receptor for SARS-CoV2. A study, DARE-19, was carried out to unveil the effects of SGLT-2 inhibitor treatment on comorbid disease complications and concomitant COVID-19 outcomes and demonstrated no statistical significance. However, the need for further studies is essential to provide conclusive clinical findings.
\end{abstract}

Keywords COVID-19 $\cdot$ ACE-2 $\cdot$ SGLT-2 $\cdot$ Renin angiotensin aldosterone system · DARE-19 $\cdot$ Dapagliflozin

\section{Abbreviations}

ACE-1 Angiotensin converting enzyme 1

ACE-2 Angiotensin converting enzyme 2

AMPK Adenosine monophosphate-activated protein kinase

Ang II Angiotensin II

Ang (1-7) Angiotensin (1-7)
COVID-19 Severe acute respiratory syndrome coronavirus 2

CVD Cardiovascular diseases

MAPK Mitogen-activated protein kinases

RAAS Renin angiotensin aldosterone system

SIRT1 Sirtuin 1

SGLT-2 Sodium-glucose co-transporter 2

T2DM Type 2 diabetes mellitus

The presence of comorbidities such as cardiovascular diseases (CVD), diabetes mellitus and/or renal impairment have been associated with poor prognosis in patients infected with COVID-19 [1, 2]. Numerous studies demonstrate parallels between CVD, type 2 diabetes mellitus (T2DM) and COVID-19 pathology, which accentuate pre-existing complications in patients infected with COVID-19 and potentially exacerbate the infection course $[2,3]$. A recent study elucidated that the cross-talk between the renin-angiotensin-aldosterone system (RAAS) and mitogen activated kinase pathways contributes to the exacerbation of COVID19 infection in patients with both CVD and diabetes [3]. 
From multiple ongoing studies evaluating the adjuvant role of several anti-diabetics, sodium-glucose co-transporter-2 (SGLT-2) inhibitors have garnered substantial attention owing to their efficacy in reducing the severity of cardiorenal diseases $[4,5]$. Nonetheless, the pertinent question holds as to whether SGLT-2 inhibitors provide protective effects in patients with COVID-19 or whether they aggravate the underlying pathophysiologic conditions. To address this question, DARE-19 (Dapagliflozin in respiratory failure in patients with COVID-19), a pragmatic investigatorsponsored, collaborative, multi-center, randomized, doubleblind, placebo-controlled phase III clinical trial, was carried out to unveil the effects of SGLT-2 inhibitor treatment on comorbid disease complications and concomitant COVID19 outcomes in patients exhibiting mild to moderate symptoms on admission [6].

The DARE-19 study aimed at evaluating the efficacy of the SGLT-2 inhibitor, dapagliflozin, in the prevention of the incidence of major events, all-cause mortality and in improving clinical recovery in patients hospitalized with COVID-19 [6]. The study also assessed the safety profile of dapagliflozin and evaluated two primary endpoints that constituted: (1) time to first occurrence of a new major or worsened cardiometabolic event, organ dysfunction, or time to death from any cause at any time during index hospitalization and the 30-day treatment period, (2) the hierarchical composite endpoint ranking change in clinical status during the entire duration of the treatment period [6]. To contextualize the insights gleaned from this trial, it is imperative to deconstruct the rationale behind SGLT-2 inhibitor use and assess its effects on the RAAS cascade that either ameliorate or amplify the extant pathologic conditions in patients infected with COVID-19.

\section{Cardio- and reno-protective role of SGLT-2 inhibitors}

SGLT-2 is a glucose transporter that is responsible for approximately $90 \%$ of the plasma glucose reabsorption in the kidney [7]. Expression and activity of SGLT-2 are significantly up-regulated in T2DM [7]. SGLT-2 inhibitors, including empagliflozin and dapagliflozin, are a new class of oral anti-diabetic agents specifically aimed to suppress glucose reabsorption in the proximal renal tubule and increase urinary glucose excretion [7]. Besides the noticeable improvement in glycemic control, SGLT-2 inhibitors provide potential additional benefits including reno- and cardio-protective effects by inducing natriuresis, osmotic diuresis, and uricosuria [7]. Empagliflozin has been demonstrated to contribute to cardio- and reno-protective effects by inducing a transcriptional paradigm that recapitulated a state of fasting via upregulation of the SIRT1/AMPK pathway and
Table 1 Cardiorenal protective effects of empagliflozin

\begin{tabular}{ll}
\hline A & Reduced oxidative stress \\
B & Enhanced mitochondrial structure and function \\
C & Minimization of coronary microvascular injury \\
D & Decreased inflammation \\
E & Improved contractile performance \\
F & Reduced glomerular and tubular inflammation and injury \\
\hline
\end{tabular}

concomitant suppression of the Akt/mTOR signaling pathway. This in turn reduced oxidative stress, normalized the mitochondrial structure and function, minimized coronary microvascular injury, suppressed inflammation, improved contractile ability and attenuated the development of cardiomyopathy [8] (Table 1). Another study elucidated that the amplified interplay of the SIRT1 and AMPK also orchestrated the action of dapagliflozin to ameliorate glomerular and tubular inflammation and injury to preclude development of nephropathy [9].

\section{Alterations of the RAAS cascade in CVD}

The RAAS cascade includes the activator and the inhibitory pathway. The activator pathway results in vasoconstriction and pro-inflammatory responses and is directed by angiotensin converting enzyme 1 (ACE-1), an enzyme that produces angiotensin II (AngII). AngII is an active peptide that causes the vasoconstriction, oxidative stress and inflammation. The inhibitory pathway results in vasodilation and anti-inflammation and is directed by angiotensin converting enzyme 2 (ACE-2), a peptidase which competes with ACE-1 and deactivates AngII by metabolizing it to angiotensin III [10]. It has been demonstrated that enhanced RAAS activity underlies CVD conditions and favors increased AngII levels [10, 11]. In this context, activation of the type- 1 angiotensin-II receptor $\left(\mathrm{AT}_{1} \mathrm{R}\right)$ by AngII mediates various intracellular pathways that orchestrate endothelial dysfunction, cardiac and vascular remodeling, and atherosclerosis. Furthermore, $\mathrm{AT}_{1} \mathrm{R}$ stimulation also facilitates activation of protein kinases such as mitogen-activated protein kinases (MAPK). Contrariwise, repression of MAPK cascades mediated by angiotensin (1-7) [Ang (1-7)] produced via ACE-2-catalyzed cleavage of AngII, decreases inflammation and maintains a well-balanced RAAS activity between ACE-2/ Ang (1-7) and ACE-1/AngII pathway. Correspondingly, recent studies showed that phosphotyrosine phosphatase activation and MAPK cascade suppression facilitated by Ang (1-7)-mediated type-2 angiotensin-II receptor $\left(\mathrm{AT}_{2} \mathrm{R}\right)$ activation confers a protective effect against CVD symptoms $[12,13]$. Thus, activation of the RAAS cascade in the 
presence of ACE/ARB inhibition will favor the Ang(1-7) and the activation of the $\mathrm{AT}_{2} \mathrm{R}$ [13].

\section{The interplay between RAAS, CVD and COVID-19}

In CVD patients, ACE-2 serum levels have been demonstrated to be elevated, which is further increased when there is an active COVID-19 infection, putting them at risk of severe COVID-19 symptoms [14]. It has also been demonstrated that the SARS-CoV strain contributes to myocardial and pulmonary infections in the presence of discernible reductions in ACE-2 expression levels [15]. This, would therefore, attenuate the protective effects of the ACE-2/ Ang (1-7) pathway, thus leading to perturbation of the RAAS-ACE-2 homeostasis and cause potential deterioration of both respiratory symptoms and cardiovascular complications. Similarly, in vivo, it has been demonstrated that pulmonary failure increases following viral spike glycoprotein injection due to the downregulation of ACE-2 expression levels [16]. Clearly, ACE-2 plays a role in mediating SARS-CoV in the heart but whether it is downregulated or upregulated requires further studies.

\section{SGLT-2 inhibitors influence RAAS in the light of COVID-19}

The safety of SGLT-2 inhibitors in patients infected with COVID-19 was questioned patients due to pre-clinical studies which have demonstrated that SGLT-2 inhibitors can cause transient activation of systemic RAAS, and that intrarenal RAAS is sometimes activated to compensate for the $\mathrm{Na}^{+}$and water loss, particularly after the initiation of treatment [17]. Chronic use of the SGLT-2 inhibitor did not result in increased activity of the RAAS pathway [18]. The activation of the RAAS cascade increases the production of AngII resulting in inflammation. However, it seems that while SGLT-2 inhibitors deplete the circulating volume resulting in activation of the RAAS cascade, it exerts an organ protective effect in the presence of ACE.ARB inhibitors that prevent the activation of $\mathrm{AT}_{1} \mathrm{R}$ resulting in the activation of $\mathrm{AT}_{2} \mathrm{R}$ [19]. Whether RAAS activity is upregulated in patients who have recently been prescribed SGLT-2 inhibitors versus those who have been on SGLT-2 inhibitors over a long period remains unknown. How such findings correlate to the effects of SGLT2 inhibitors in patients with COVID-19 also remains unclear.

\section{DARE-19 study}

Evidence of the efficacy of SGLT-2 inhibitors in conferring substantive organ protection in patients infected with COVID-19 remains elusive. The Phase III DARE-19 study with 1229 patients demonstrated no statistical significance for its two primary endpoints during the 30 days follow up. The first outcome being the time to first occurrence of new or worsened organ dysfunction or death which occurred in 64 patients in dapagliflozin arm vs 80 patients in placebo group and the second outcome being hierarchical recovery outcome, which occurred in $87.5 \%$ of the dapagliflozin arm vs $85.1 \%$ in the placebo arm [20]. The need to orchestrate robust large-scale clinical trials constituting a longer duration (90-120 days) is highly warranted [21]. Evaluating the effectiveness of combination therapy involving, for example, SGLT-2 inhibitors and renin-angiotensin system blockers, in ameliorating adverse cardiorenal events in hospitalized patients with COVID-19 would serve as another prudent investigation [22]. Similarly, looking at the time in which SGLT-2 inhibitors were initiated and further in-depth analysis of the RAAS cascade would provide further insight into the role of RAAS in contributing to the clinical outcome of patients using SGLT-2 inhibitors and infected with COVID-19. Although the data gleaned from the DARE-19 trial demonstrated no disproportionate benefit or harm in terms of cardioprotection, it is substantiated dapagliflozin's well-established safety and tolerability profile. Hence, the findings of this trial should not entirely be discussed with negative connotations, but rather with a positive perspective, as they underscored that administration of SGLT-2 inhibitors would not worsen prognosis of COVID-19, although it would not necessarily confer discernible cardioprotection.

Funding This project was supported by Qatar University Student Grant No: QUST-2-CPH-2020-16. "The funders had no role in study design, data collection, and analysis, decision to publish, or preparation of the manuscript."

Data availability Not applicable.

Code availability Not applicable.

\section{Declarations}

Conflict of interest The authors have no conflicts of interest to declare that are relevant to the content of this article.

Ethical approval This article does not contain any studies with human participants or animals performed by any of the authors.

Consent to participate Not applicable.

Consent for publication Not applicable. 


\section{References}

1. Gupta K, Kunal S (2020) SGLT-2 inhibitors as cardioprotective agents in COVID-19. Heart Lung 49(6):875-876

2. Ejaz H, Alsrhani A, Zafar A, Javed H, Junaid K, Abdalla AE et al (2020) COVID-19 and comorbidities: deleterious impact on infected patients. J Infect Public Health 13(12):1833-1839

3. Feldman EL, Savelieff MG, Hayek SS, Pennathur S, Kretzler M, Pop-Busui R (2020) COVID-19 and diabetes: a collision and collusion of two diseases. Diabetes 69(12):2549

4. Neuen BL, Young T, Heerspink HJL, Neal B, Perkovic V, Billot L et al (2019) SGLT2 inhibitors for the prevention of kidney failure in patients with type 2 diabetes: a systematic review and metaanalysis. Lancet Diabetes Endocrinol 7(11):845-854

5. Zelniker TA, Wiviott SD, Raz I, Im K, Goodrich EL, Bonaca MP, Mosenzon O, Kato ET, Cahn A, Furtado RH, Bhatt DL (2019) SGLT2 inhibitors for primary and secondary prevention of cardiovascular and renal outcomes in type 2 diabetes: a systematic review and meta-analysis of cardiovascular outcome trials. The Lancet 393(10166):31-39

6. Kosiborod M, Berwanger O, Koch G, Martinez F, Mukhtar O, Verma $S$ et al (2021) Effects of dapagliflozin on prevention of major clinical events and recovery in patients with respiratory failure because of COVID-19: design and rationale for the DARE19 study. Diabetes Obes Metab 23(4):886-896

7. Zou H, Zhou B, Xu G (2017) SGLT2 inhibitors: a novel choice for the combination therapy in diabetic kidney disease. Cardiovasc Diabetol. https://doi.org/10.1186/s12933-017-0547-1

8. Zhou H, Wang S, Zhu P, Hu S, Chen Y, Ren J (2018) Empagliflozin rescues diabetic myocardial microvascular injury via AMPK-mediated inhibition of mitochondrial fission. Redox Biol 1(15):335-346

9. Terami N, Ogawa D, Tachibana H, Hatanaka T, Wada J, Nakatsuka A, Eguchi J, Horiguchi CS, Nishii N, Yamada H, Takei K (2014) Long-term treatment with the sodium glucose cotransporter 2 inhibitor, dapagliflozin, ameliorates glucose homeostasis and diabetic nephropathy in $\mathrm{db} / \mathrm{db}$ mice. PloS one 9(6):e100777

10. Mori J, Oudit G, Lopaschuk G (2020) SARS-CoV-2 perturbs the renin-angiotensin system and energy metabolism. Am J PhysiolEndocrinol Metab 319(1):E43-E47

11. Wehbe Z, Hammoud S, Soudani N, Zaraket H, El-Yazbi A, Eid A (2020) Molecular insights into SARS COV-2 interaction with cardiovascular disease: role of RAAS and MAPK signaling. Front Pharmacol. https://doi.org/10.3389/fphar.2020.00836

12. Azushima K, Morisawa N, Tamura K, Nishiyama A (2020) Recent research advances in renin-angiotensin-aldosterone system receptors. Curr Hypertens Rep 22(3):22
13. Kawanami D, Matoba K, Takeda Y, Nagai Y, Akamine T, Yokota T et al (2017) SGLT2 inhibitors as a therapeutic option for diabetic nephropathy. Int J Mol Sci 18(5):1083

14. Chen L, Li X, Chen M, Feng Y, Xiong C (2020) The ACE2 expression in human heart indicates new potential mechanism of heart injury among patients infected with SARS-CoV-2. Cardiovasc Res 116(6): 1097-1100

15. Oudit GY, Kassiri Z, Jiang C, Liu PP, Poutanen SM, Penninger JM, Butany J (2009) SARS-coronavirus modulation of myocardial ACE2 expression and inflammation in patients with SARS. Eur J Clin Invest 39(7):618-625

16. Kuba K, Imai Y, Rao S, Gao H, Guo F, Guan B, Huan Y, Yang P, Zhang Y, Deng W, Bao L (2005) A crucial role of angiotensin converting enzyme 2 (ACE2) in SARS coronavirus-induced lung injury. Nat Med 11(8):875-879

17. Cherney DZ, Perkins BA, Soleymanlou N, Xiao F, Zimpelmann J, Woerle HJ, Johansen OE, Broedl UC, Von Eynatten M, Burns KD (2014) Sodium glucose cotransport-2 inhibition and intrarenal RAS activity in people with type 1 diabetes. Kidney Int 86(5): 1057-1058

18. Li L, Konishi Y, Morikawa T, Zhang Y, Kitabayashi C, Kobara $\mathrm{H}$ et al (2018) Effect of a SGLT2 inhibitor on the systemic and intrarenal renin-angiotensin system in subtotally nephrectomized rats. J Pharmacol Sci 137(2):220-223

19. Muskiet MH, van Raalte DH, van Bommel EJM, Smits MM, Tonneijck L (2015) Understanding Empa-Reg outcome. Lancet Diabetes Endocrinol 3(12):928-9

20. COVID-19, U., (2021). Update on the DARE-19 Phase III trial for Farxiga in COVID-19. [online] Astrazeneca.com. https://www. astrazeneca.com/media-centre/press-releases/2021/update-onfarxiga-covid-19-dare-19-phase-iii-trial.html\#: :text=In\%20Aug ust $\% 202020 \% 2 \mathrm{C} \% 20$ results $\% 20$ from,and $\% 20$ without $\% 20 \mathrm{~T} 2 \mathrm{D} \%$ 20versus\%20placebo. Accessed 9 July 2021.

21. TCTMD.com. (2021). Dapagliflozin No Help, but No Harm, in Hospitalized COVID-19 Patients. [online]. https://www.tctmd. com/news/dapagliflozin-no-help-no-harm-hospitalized-covid19-patients. Accessed 9 July 2021.

22. Zhao L, Zhang M, Zhan Z, Qiu M (2021) Does combination therapy with SGLT2 inhibitors and renin-angiotensin system blockers lead to greater reduction in cardiorenal events among patients with type 2 diabetes. Front Cardiovasc Med. https://doi.org/10.3389/ fcvm.2021.679124

Publisher's Note Springer Nature remains neutral with regard to jurisdictional claims in published maps and institutional affiliations. 